\title{
Potential HNF1A-AS1/miR-214/INHBA signal regulation mechanism in colorectal cancer based on bioinformatics analysis and validation
}

\section{Xuan Zhang}

Yunnan Cancer Hospital, The Third Affiliated Hospital of Kunming Medical University

\section{Tao Wu}

Yunnan Cancer Hospital, The Third Affiliated Hospital of Kunming Medical University

\section{Rong Ding}

Yunnan Cancer Hospital, The Third Affiliated Hospital of Kunming Medical University

\section{Rujia Qin}

Yunnan Cancer Hospital, The Third Affiliated Hospital of Kunming Medical University

\section{Yongchun Zhou}

Yunnan Cancer Hospital, The Third Affiliated Hospital of Kunming Medical University

\section{Xiaoxiong Wang}

Yunnan Cancer Hospital, The Third Affiliated Hospital of Kunming Medical University

\section{ZhongJun Shang}

Yunnan Cancer Hospital, The Third Affiliated Hospital of Kunming Medical University

\section{GuoYu Li}

Yunnan Cancer Hospital, The Third Affiliated Hospital of Kunming Medical University

Chao Dong

Yunnan Cancer Hospital, The Third Affiliated Hospital of Kunming Medical University

Renfang Yang

Yunnan Cancer Hospital, The Third Affiliated Hospital of Kunming Medical University Jinsha Li

Yunnan Cancer Hospital, The Third Affiliated Hospital of Kunming Medical University

\section{Yunfeng Li ( $\square$ zhangxuan66@kmmu.edu.cn )}

Yunnan Cancer Hospital, The Third Affiliated Hospital of Kunming Medical University

\section{Research Article}

Keywords: Colorectal Cancer, LncRNA HNF1A-AS1, miR-124, INHBA, TGF- $\beta$ /Smad

Posted Date: January 14th, 2022

DOI: https://doi.org/10.21203/rs.3.rs-1170130/v1 
License: (c) (i) This work is licensed under a Creative Commons Attribution 4.0 International License. Read Full License 


\section{Abstract}

Preceding studies have identified that noncoding RNA plays a significant role in the occurrence and development of tumors. Colorectal cancer (CRC) has attracted increasing attention due to its high incidence and mortality rate. Based on Cancer Genome Atlas (TCGA) database analysis, it was found that compared with normal tissues, HNF1A-AS1 and INHBA were highly expressed in CRC tissues; miR-214 was relatively low expressed, and it is predicted to specifically target the3' untranslated region (3'UTR region) of INHBA. Besides, the result was consistent with the quantitative reverse transcription PCR (RTqPCR) verification results of $17 \mathrm{CRC}$ cases and adjacent tissues collected clinically. Western Blot (WB) manifested that INHBA protein was highly expressed in CRC tissues, which was consistent with the results of CRC cell lines (HT29, SW480). Immunohistochemical (IHC) staining demonstrated that INHBA protein was brownish yellow, overwhelming majority of INHBA protein were located in the cytoplasm, and expression level was significantly higher than that in adjacent tissues. Based on previous studies, the biological process of INHBA-mediated TGF- $\beta /$ Smad signaling pathway inducing the proliferation and invasion of CRC has been partially confirmed, but the upstream signaling molecules and mechanisms which regulating INHBA remain unclear. Herein, benefiting from bioinformatics, preliminary experimental results and previous research, they provide basis for the follow-up study on the regulation of HNF1AAS1/miR-214/INHBA signal axis in CRC.

\section{Introduction}

Colorectal cancer (CRC) is one of the most common gastrointestinal malignancies. In 2020, the incidence rate of CRC ranked the third and the mortality rate ranked second(1). Besides, China cancer statistics report showed the incidence and mortality of $\mathrm{CRC}$ ranked third and fifth respectively among all malignant tumors, with 376,000 new cases and 191,000 deaths in 2015 (2). It is pointed out that CRC is a heterogeneous disease, regulated by numerous factors and with intricated biological process, and its occurrence and development mechanism remains to be elucidated (3). In recent years, epigenetic and transcriptome changes have attracted the attention of researchers owing to their significant role in tumors development $(4,5)$.

MiRNA is a noncoding single-stranded RNA (19-25nt) derived from endogenous hairpin structure transcripts. It is a noncoding small molecular RNA that regulate the expression of other genes, which can pass through the 3'UTR region of its target gene mRNA sequence or coding region specific base complementary pairing combination causes the degradation of target mRNA or the inhibition of target gene translation process, so as to regulate the expression of target gene at the post transcriptional level $(6,7)$. Studies have shown that miRNAs play an crucial role in tumor growth, apoptosis, invasion and metastasis. They restrain the expression of target genes by combining with the translation of target genes, and then participate in the regulation of $30 \%$ human protein expression, tumorigenesis, development and metastasis (8). And long noncoding RNA (LnCRNA) is also an irreplaceable role in the subsequent research progress of human tumors. As a competitive endogenous RNA (ceRNA), LncRNA 
performs a vital role in the tumorgenesis by binding miRNA to regulate the expression of target genes. $(9$, 10).

Inhibin is a homodimer or heterodimer consisting of $a, \beta A$ and $\beta B$ subunits, which are called inhibins $a$ (INHA), inhibin $\beta A$ (INHBA), inhibin $\beta B$ (INHBB) subunit gene (11). INHBA gene is located on chromosome 7p15-p14 and consists of two exons (11). As a member of transforming growth factor beta (TGF- $\beta$ ) superfamily, INHBA gene participate in the occurrence and development of tumors by regulating TGF$\beta /$ Smad signaling pathway, and abnormal expression of INHBA gene is associated with poor prognosis of cancers (12). Preceding researchers have illustrated that INHBA gene also promote the invasion and metastasis of cancers by inducing epithelial-mesenchymal transition (EMT), promoting angiogenesis and changing epigenetic mechanisms.

Based on the transcriptome data of 473 colon cancer tissues and 41 adjacent tissues collected in the cancer genome atlas (TCGA) database, our research team found that INHBA was highly expressed in cancer tissues, and the expression of INHBA significantly positively correlated with the T stage of tumor, that is, the higher the expression level of INHBA, the later the T stage of patients $(P<0.05)$. Meanwhile, the survival curve analysis of gene expression profiling interactive analysis (GEPIA) database manifested that the expression level of INHBA was negatively correlated with the survival rate of colon cancer patients, that is, the higher the expression level of INHBA, the lower the survival rate of colon cancer patients. The results of quantitative reverse transcription PCR (RT-qPCR) and Western Blot of 17 cases of CRC and adjacent tissues also identified that the mRNA and protein levels of INHBA were also highly expressed. Besides, immunohistochemical (IHC) staining also demonstrated that INHBA protein was brownish yellow, which were principally located in the cytoplasm, and the expression level of INHBA protein was significantly higher than that in adjacent tissues. The previous studies showed that HNF1AAS1 and miR-214 were closely related to the progression of CRC, and bioinformatics confirmed that miR214 specifically targets the 3'UTR of INHBA gene. Further analysis indicated that the expression of HNF1A-AS1 and miR-214 was consistent in TCGA database and experiment. In addition, previous studies have shown that INHBA gene can regulate TGF- $\beta /$ Smad signaling pathway and promote the occurrence and development of tumors $(12,13)$. Therefore, we speculate that miR-214 regulate the activity of TGF$\beta /$ Smad signal axis by targeting INHBA, thereby inhibiting the proliferation and metastasis of CRC.

\section{Materials And Methods}

\section{Clinical samples collection}

We collected 17 CRC samples and para-cancer tissues from Yunnan Cancer Hospital/The Third affiliated Hospital of Kunming Medical University. The age of the CRC patients ranged from 46 to 69 years, including 13 males (median age was 62) and 4 females (median age was 65). All tumor and adjacent tissues removed from the patient were immediately placed in liquid nitrogen and transported to the laboratory within 2 hours for storage in $-80^{\circ} \mathrm{C}$ degree refrigerator. Written informed consent was obtained from all participants prior to their enrollment of the study. The protocols for the present study were 
approved by the Medical Institutional and Clinical Research Ethics Committee of Yunnan Cancer Hospital, the Third Affiliated Hospital of Kunming Medical University (refers to the same entity) in accordance with the "Declaration of Helsinki" on the ethical principles for medical research involving human subjects.

\section{Analysis of HNF1A-AS1 in diverse human tumor}

Preceding studies have manifested that HNF1A-AS1 is a potential long noncoding RNA to regulate tumor progression. Using the TCGA (https:// cancer.genome.nih.gov/) database, we analyzed the expression of HNF1A-AS1 in 33 different categories tumors and adjacent tissues. In addition, we analyzed the expression of HNF1A-AS1 in colon cancer and rectal cancer tissues and corresponding para-cancer tissues and its association with overall survival(OS). And we further verified differences in HNF1A-AS1 expression in colon cancer and rectal cancer in relative para-cancer tissues in the two databases.

\section{Differential expression of HNF1A-AS1/miRNAs}

Firstly, we analyzed the expression difference of HNF1A-AS1 in 477 tumors and 41 adjacent tissues in TCGA database. Then, the distribution of 125 immune related genes associated with HNF1A-AS1 in tumor and normal tissues was analyzed. Subsequently, the differentially expressed miRNAs (DEMs) from tumors and adjacent tissues in the database were analyzed by volcano plots. Finally, in order to identify the correlation between HNF1A-AS1 and differentially expressed miRNAs, TargetScan was used to predict and construct its association.

\section{Gene Ontology (GO) analysis}

To explore the biological function of 125 immune related genes associated with HNF1A-AS1, the R package 'clusterprofiler' R package was used to analyse the Gene Ontology (GO) function and Kyoto Encyclopedia of Genes and Genomes (KEGG) pathway enrichment. P-value $<0.05$ was considered statistically significant.

\section{Association between HNF1A-AS1 and differential miRNAs}

The relative expression of miRNA which tightly linked with HNF1A-AS1 was detected by RT-qPCR in clinical CRC and para-cancer tissues. Subsequently, the association between differentially expressed miRNAs and HNF1A-AS1 was analyzed.

\section{Target gene analysis of hsa-miR-214}

The miRDB, miRWalk, and TargetScan databases were utilized to predict the target genes of hsa-miR-214. RT- qPCR was sued to detect the relative expression level of target genes located in overlapping region. Meanwhile, the relative expression levels of INHBA in CRC and para-cancer tissues were analyzed by TCGA database. In addition, the expression of INHBA at different stages of CRC and its association with disease-free survival (DFS) and OS were analyzed. 


\section{Real time quantitative PCR}

Samples were homogenized with Trizol reagent (tarkara), and total RNA was extracted according to the kit instructions. After quantification by NanoDrop 2000 (Tarkara), $200 \mathrm{ng}$ total RNA was utilized by ReverTra Ace qPCR RT Kit (Tarkara) for reverse transcription according to the instructions. THUNDERBIRD SYBR ${ }^{8}$ qPCR Mix (Tarkara) was used for RT-qPCR analysis. CFX96 Touch Real-Time PCR Detection System (Bio-RAD) was used for RT-qPCR. GAPDH was used as internal reference when determining the contents. Corresponding primer sequences are shown in supplementary material.

\section{Western blot experiment}

Cell lysis buffer solution was added into the sample, and total protein extract was obtained after repeated suspension and centrifugation at 4 degrees. Protein concentration in the extract was detected by Bradford method. SDS-PAGE was used for protein electrophoresis, followed by $300 \mathrm{~mA}$ constant current for $1 \mathrm{~h}$ to transfer the protein onto PVDF membrane. After sealing, primary antibody was added and shake it overnight at $4^{\circ} \mathrm{C}$. After washing the membrane, add secondary antibody and incubate at $37^{\circ} \mathrm{C}$ for $2 \mathrm{~h}$. Then add luminous solution and perform tablet pressing, exposure and fixing. Image $J$ analysis was used to obtain the image and determine the integral optical density of protein bands, and GAPDH protein was selected as the internal reference.

\section{Immunohistochemistry assay}

Immunohistochemistry (IHC) assay was performed according to the previous process (14). Briefly, Paraffin sections were placed at $60^{\circ} \mathrm{C}$ for $2 \mathrm{~h}$, then washed by dimethylbenzene solution, ethanol, and TBS in sequence. After antigen retrieved with citrate buffer solution, endogenous peroxidase was blocked by $1 \%$ hydrogen peroxide. Tissues were incubated with primary antibody overnight at $4^{\circ} \mathrm{C}$. Subsequently, the sections were incubated with HRP-labeled goat anti-rabbit immunoglobulin G (1:100, Abcam). Sections were visualized by diaminobenzidine. Then sections were counterstained with hematoxylin and washed by dimethylbenzene solution and ethanol. At last, sealed the sections with neutral balsam. The results of immunohistochemical staining were analyzed with optical microscope.

\section{Statistical analysis}

SPSS 23.0 was utilized to analyze the data, and graphpad prism 8.0 software was used for statistical mapping. The measurement data are expressed by mean standard deviation (mean \pm standard deviation, \pm s). T-test or analysis of variance (ANOVA) was used to compare the group comparison of measurement data subject to Gaussian Distribution, otherwise the nonparametric rank sum test was used. Chi-square test was used for counting data. $P<0.05$ was considered statistically significant.

\section{Results}

HNF1A-AS1 is highly expressed in colon cancer and rectal cancer tissues compared with para-cancer tissues 
HNF1A-AS1 is expressed differently in different human tumor tissues (Figure 1A), but it is highly expressed in colon cancer and rectal cancer (Figure 1B). Although the expression level of HNF1A-AS1 in colon cancer and rectal cancer was correlated with the OS, there was no statistical difference (Figure 1CD). In addition, we found that in the database of colon cancer and rectal cancer, HNF1A-AS1 was highly expressed in cancer tissues compared with para-cancer tissues, and the difference was statistically significant $(P<0.05)$ (Figure 1E-F).

\section{HNF1A-AS1 related miRNAs analysis}

The hierarchical cluster analysis diagram of Figure 2A shows the expression difference of AS1 in 477 tumors and 41 adjacent tissues. Similarly, Figure 2B demonstrated differences in the expression of 125 immune-related genes associated with HNF1A-AS1. With the cut-off value of adj.P.Val $\leq 0.05$, compare with the control, we obtained 2027 up-regulated genes and 1404 down-regulated genes (Figure 2C), Meanwhile, based on TargetScan data, we found 65 miRNAs that may bind to HNF1A-AS1 (Figure 2D). Moreover, GO and KEGG analysis of 125 genes are showed related to immunity include CD4-positive, alpha-beta $\mathrm{T}$ cell differentiation involved in immune response, T-helper 17 type immune response, T-helper 1 type immune response, intestinal immune network for IgA production (Figure 3A-B).

\section{MiR-214 was low expressed in CRC compared with para-cancer tissues}

RT-qPCR results identified that compared with para-cancer tissues, hsa-miR-1252-3p, hsa-miR-214, hsamiR-3907, hsa-miR-4688-5p, hsa-miR-10527-5p were lower expressed $(P<0.05)$, while HNF1A-AS1 was higher expressed in the CRC tissues $(P<0.05)$ (Figure 4A). Besides, the expression of HNF1A-AS1 was negatively correlated with hsa-miR-1252-3p, hsa-miR-214, hsa-miR-3907, hsa-miR-4688-5p, hsa-miR10527-5p, but there were only statistical differences in hsa-miR-214, hsa-miR-4688-5p and hsa-miR-1252$3 p(P<0.05)$ (Figure 4B-F).

\section{The target gene INHBA of hsa-miR-214 is highly expressed in CRC}

The miRDB, miRWalk, and TargetScan databases predicted four overlapping genes of miR-214 target genes, namely CXCR5, GFRA1, SNAP25 and INHBA (Figure 5A). RT-qPCR results showed that compared with adjacent tissues, CXCR5, GFRA1, and SNAP25 genes were lower expressed in CRC, while INHBA gene was highly expressed in CRC $(P<0.001)$ (Figure 5B-E).

\section{MiRNA-214-5p is positively correlated with INHBA in colon cancer}

The specific binding sites of INHBA-3'UTR and miR-214-5p were identified by starBase, and the results indicated that INHBA might be the target gene of miR-214-5p, that is, miR-214-5p might regulate the expression of INHBA gene through post-transcriptional mode (Figure 6A). Through enrichment analysis of INHBA gene, it was found that miR-214-5p may be one of the key miRNAs regulated upstream of INHBA (Figure 6B). Analysis of 450 colon cancer samples by TCGA database indicates that miRNA-214-5p is positively correlated with INHBA (Figure 6C). TCGA database was used to analyze the miRNAs data of 
261 CRC tissues and control normal tissues, and the results identified that miRNA-214-5p was low expressed in tumor tissues compared with the adjacent tissues $(P<0.01)$ (Figure 6D).

\section{The expression of INHBA gene is associated with the progression of CRC}

According to the transcriptome data of $473 \mathrm{CRC}$ and 41 adjacent tissues gathered in TCGA database, it was found that INHBA was highly expressed in cancer tissues (Figure 7A-7B), and the expression level of INHBA was positively correlated with the $T$ stage of tumor, that is, the higher the expression level of INHBA, the later the T stage of patients $(P<0.05)$ (Figure 7C). However, there was no correlation between the expression level of INHBA and the $\mathrm{N}$ stage, or M stage of tumor (P[0.05)(Figure 7D-7E). The survival curve analysis of GEPIA database manifested that there was a negative correlation between the expression level of INHBA and the survival rate of CRC patients, that is, the higher the expression level of INHBA, the lower the OS and DFS of CRC patients $(P<0.05)$ (Figure 7F-7G). The prognostic risk score model of CRC was constructed based on INHBA gene, and the area under the curve (AUC): 0.996 (95\% Cl: $0.993 \sim 1.000, P<0.05)$, indicating that INHBA gene has high accuracy in predicting the prognosis of CRC (Figure 7H).

\section{INHBA is highly expressed in CRC based on experimental verification}

IHC staining was utilized to analyze the distribution and expression of INHBA in cancer and para-cancer tissues of 17 patients with CRC. Positive INHBA protein was brown-yellow, principally located in the cytoplasm. The results demonstrated that the expression level of INHBA protein in cancer tissues (Figure $\mathbf{8 A}, \mathbf{8 C}$ ) was significantly higher than that in paired para-cancer tissues (Figure $\mathbf{8 B}, \mathbf{8 D}$ ). RT-qPCR and Western Blot results indicated that the transcription and protein level of INHBA in CRC tissues were higher than those in adjacent tissues $(P<0.05$ )(Figure $8 \mathrm{E}-\mathbf{G}$ ). In addition, INHBA was highly expressed in $\mathrm{CRC}$ cell lines (HT29 and SW480) compared with normal intestinal epithelial cells (NCM460) (Figure 8H). All original, uncropped, full-length gels and blots are presented in the supplementary Information file.

\section{Discussion}

Previous studies have shown that INHBA gene can participate in the occurrence and development of gastric cancer by regulating TGF- $\beta$ /Smad signaling pathway (13), and it can also promote the invasion and metastasis of breast cancer, hepatocellular carcinoma and non-small cell lung cancer by inducing EMT (15), promoting angiogenesis (16) and changing epigenetics (17). TGF- $\beta$ /Smad signaling pathway is a tumor inhibitory pathway mediated by membrane serine/threonine kinase receptor, and its main components include TGF- $\beta$ Superfamily, TGF- $\beta$ Receptors, Smad Protein Family, and its nuclear transcription regulators and abnormalities in any component of this pathway can lead to tumorigenesis (18). A recent study found that knockdown of INHBA can significantly inhibit the expression of TGF- $\beta$ signaling pathway-related proteins in CRC cells (19).

However, the role of INHBA-mediated TGF- $\beta$ /Smad signaling axis in the development and progression of CRC remains to be further studied. TCGA database was used to analyze the expression of INHBA in CRC 
and adjacent tissues, and the results demonstrated that INHBA was highly expressed in cancer tissues, and the expression level of INHBA was positively correlated with the T stage of tumor, that is, the higher the level of INHBA, the later the T stage of patients $(P<0.05)$. In order to verify the credibility of the database results, we collected clinical CRC tissue samples and tested the expression level of INHBA in the cancer tissue and the adjacent tissue by IHC, RT-qPCR and Western Blot respectively. The results of the three groups confirmed that the expression level of INHBA in the CRC tissues was significantly higher than that in the control adjacent tissue. In addition, compared with normal intestinal epithelial cells (NCM460), INHBA protein levels were highly expressed in CRC cell lines (HT29 and SW480). Finally, survival curve analysis of GEPIA database identified that INHBA expression level was negatively correlated with OS and DFS in CRC patients. Therefore, we speculate that whether INHBA, as a tumor suppressor gene, also promotes CRC progression through regulating TGF- $\beta / S$ mad signaling pathway, which will be further verified in subsequent studies.

Preceding studies have confirmed that miR-214 can play a tumor suppressive role in endometrial cancer, breast cancer and other tumors through diverse molecular mechanisms $(20,21)$. Besides, studies also suggested that miR-214 can be used as an crucial tumor suppressor in CRC (22). For example, miR-214 can inhibit the proliferation and metastasis of CRC by targeting the PLAGL2-MYH9 axis (23), while as miR-214 can also inhibit LIVIN and NF-KB signaling pathway, which plays an anti-cancer role. Therefore, it is speculated that miR-214 may inhibit the proliferation and metastasis of CRC. MiRNA data of 261 colon cancer tissue samples and control samples collected from TCGA database were analyzed, and the results showed that miR-214 was lower expressed in tumor tissues than in normal controls $(P<0.01)$. Other studies have found that miR-214 can inhibit TGF- $\beta$ mediated activation of pancreatic stellate cells and the occurrence of pancreatic cancer (24), but the idea that whether miR-214 can inhibit the occurrence and development of CRC by down-regulating the activity of TGF- $\beta / S m a d$ signaling axis remains to be further verified. Bioinformatics enrichment analysis found that miR-214 may be one of the key miRNAs regulated upstream of INHBA, and it was predicted that INHBA-3 'UTR specifically binds to miR-214 .

LncRNA has been proved to regulate various biological processes such as cell proliferation, apoptosis, invasion and metastasis, which provides a new perspective for our diagnosis and treatment of cancer(25). HNF1A-AS1 is highly expressed in diverse tumors, promoting the proliferation of triplenegative breast cancer (26), hepatocellular carcinoma (27) and glioma (28); proliferation, migration and invasion of osteosarcoma (29); proliferation, apoptosis, chemoresistance of non-small-cell cancer (NSCLC) (30); proliferation, migration, EMT of oral squamous cell carcinoma (31); invasion, metastasis, angiogenesis and lymphangiogenesis of gastric cancer (32); metastasis and invasion of bladder cancer (33); proliferation, migration and invasion of oesophageal adenocarcinoma (34). Our results from TCGA database and clinical samples showed that HNF1A-AS1 was highly expressed in CRC. In addition, there was a negative correlation between the expression of HNF1A-AS1 and miR-214 $(P<0.05)$.

In summary, our preliminary results suggest that HNF1A-AS1/miR-214 /INHBA/TGF- $\beta /$ Smad signaling axis may be a potential mechanism for regulating $C R C$, which will be verified later. 


\section{Declarations}

\section{Acknowledgement}

Not applicable.

\section{Funding}

This study was supported by the National Natural Science Foundation of China (82060542), and the Scientific Research Fund of Yunnan Provincial Education Department(2022J0227).

\section{Availability of data and materials}

Publicly available datasets were analyzed in this study. The datasets generated for this study can be found here: The Cancer Genome Atlas (TCGA) (https://portal.gdc.cancer.gov/), Gene Expression Profiling Anteractive Analysis (GEPIA)(http://gepia.cancerpku.cn),miRDB(http://mirdb.org),miRWalk(http://mirwalk.umm.uni-heidelberg.de/), targetscan(http://www.targetscan.org/vert_80/), and starBase (http://starbase.sysu.edu.cn/) databases.

\section{Authors' contributions}

$\mathrm{XZ}, \mathrm{TW}$, and RD were responsible for study design, data acquisition, and analysis and were major contributors to writing the manuscript. RJQ, YCZ, and XXW helped to perform the data analysis. GYL, RFY, and JSL helped to perform the collection of specimens and experimental verification. ZJS, CD, and YFL were responsible for the integrity of the entire study and manuscript review. All authors contributed to the article and approved the submitted version.

\section{Ethics approval and consent to participate}

The study protocols were approved by the Medical Institutional and Clinical Research Ethics Committee of Yunnan Cancer Hospital, the Third Affiliated Hospital of Kunming Medical University (refers to the same entity). All experiments were carried out in accordance with the "Declaration of Helsinki" on the ethical principles for medical research involving human subjects. Written informed consent was obtained from all participants prior to their enrollment of the study.

\section{Competing interests}

The authors declare that they have no competing interests.

\section{References}

1. Erratum: Global cancer statistics 2018: GLOBOCAN estimates of incidence and mortality worldwide for 36 cancers in 185 countries. CA: a cancer journal for clinicians. 2020,70(4):313. 
2. Zhang S, Sun $\mathrm{K}$, Zheng R, Zeng $\mathrm{H}$, Wang $\mathrm{S}$, Chen $\mathrm{R}$, et al. Cancer incidence and mortality in China, 2015. Journal of the National Cancer Center. 2021,1(1):2-11.

3. Xu F, Tang B, Jin T, Dai C. Current status of surgical treatment of colorectal liver metastases. World journal of clinical cases. 2018,6(14):716-34.

4. Okugawa Y, Grady W, Goel A. Epigenetic Alterations in Colorectal Cancer: Emerging Biomarkers. Gastroenterology. 2015,149(5):1204-25.e12.

5. Coppedè F, Lopomo A, Spisni R, Migliore L. Genetic and epigenetic biomarkers for diagnosis, prognosis and treatment of colorectal cancer. World journal of gastroenterology. 2014,20(4):943-56.

6. Omodei D, Acampora D, Russo F, De Filippi R, Severino V, Di Francia R, Frigeri F, et al. Expression of the brain transcription factor OTXI occurs in a subset of normal germinal-center $B$ cells and in aggressive Non-Hodgkin Lymphoma. Am J Pathol. 2009,175:2609-2617.

7. Larsen K, BLutterodt MC, Møllgård K, Møller M.Expression of the homeobox genes OTX2 and OTXI in the early developing human brain. J. Histochem. Cytochem. 2010, 58: 669-678.

8. Zekri A, Youssef A, Lotfy M, Gabr R, Ahmed O, Nassar A, et al. Circulating Serum miRNAs as Diagnostic Markers for Colorectal Cancer. PloS one. 2016,11(5):e0154130.

9. Evans J, Feng F, Chinnaiyan A. The bright side of dark matter: IncRNAs in cancer. The Journal of clinical investigation. 2016,126(8):2775-82.

10. Huarte M. The emerging role of IncRNAs in cancer. Nature medicine. 2015,21(11):1253-61.

11. Laird M, Glister C, Cheewasopit W, Satchell L, Bicknell A, Knight P. 'Free' inhibin a subunit is expressed by bovine ovarian theca cells and its knockdown suppresses androgen production. Scientific reports. 2019,9(1):19793.

12. Shelling A. Mutations in inhibin and activin genes associated with human disease. Molecular and cellular endocrinology. 2012,359:113-20.

13. Chen Z, Qin L, Peng X, Hu Y, Liu B. INHBA gene silencing inhibits gastric cancer cell migration and invasion by impeding activation of the TGF- $\beta$ signaling pathway. Journal of cellular physiology. 2019,234(10):18065-74.

14. Deng S, Zhu S, Wang B, Li X, Liu Y, Qin Q, et al. Chronic pancreatitis and pancreatic cancer demonstrate active epithelial-mesenchymal transition profile, regulated by miR-217-SIRT1 pathway. Cancer letters. 2014,355(2):184-91.

15. Howley B, Hussey G, Link L, Howe P. Translational regulation of inhibin $\beta A$ by TGF $\beta$ via the RNAbinding protein hnRNP E1 enhances the invasiveness of epithelial-to-mesenchymal transitioned cells. Oncogene. 2016,35(13):1725-35.

16. Singh P, Jenkins L, Horst B, Alers V, Pradhan S, Kaur P, et al. Inhibin Is a Novel Paracrine Factor for Tumor Angiogenesis and Metastasis. Cancer research. 2018,78(11):2978-89.

17. Seder C, Hartojo W, Lin L, Silvers A, Wang Z, Thomas D, et al. Upregulated INHBA expression may promote cell proliferation and is associated with poor survival in lung adenocarcinoma. Neoplasia (New York, NY). 2009,11(4):388-96. 
18. Zhao M, Mishra L, Deng C. The role of TGF- $\beta$ /SMAD4 signaling in cancer. International journal of biological sciences. 2018,14(2):111-23.

19. He Z, Liang J, Wang B. Inhibin, beta A regulates the transforming growth factor-beta pathway to promote malignant biological behaviour in colorectal cancer. Cell biochemistry and function. 2021,39(2):258-66.

20. Fang Y, Tan M, Zhou J, Liang L, Liu X, Zhao K, et al. miR-214 inhibits epithelial-to-mesenchymal transition and metastasis of endometrial cancer cells by targeting TWIST1. OncoTargets and therapy. 2019,12:9449-58.

21. Han L, Wang H, Niu F, Yan J, Cai H. Effect miR-214 on proliferation and apoptosis of breast cancer cells by targeting survivin protein. European review for medical and pharmacological sciences. 2019,23(17):7469-74.

22. Han B, Ge Y, Cui J, Liu B. Down-regulation of IncRNA DNAJC3-AS1 inhibits colon cancer via regulating miR-214 /LIVIN axis. Bioengineered. 2020,11(1):524-35.

23. Zhou Z, Wu L, Liu Z, Zhang X, Han S, Zhao N, et al. MicroRNA-214-3p targets the PLAGL2-MYH9 axis to suppress tumor proliferation and metastasis in human colorectal cancer. Aging. 2020,12(10):9633-57.

24. Kuninty P, Bojmar L, Tjomsland V, Larsson M, Storm G, Östman A, et al. MicroRNA-199a and -214 as potential therapeutic targets in pancreatic stellate cells in pancreatic tumor. Oncotarget. 2016,7(13):16396-408.

25. Crea F, Clermont P, Parolia A, Wang $Y$, Helgason $C$. The noncoding transcriptome as a dynamic regulator of cancer metastasis. Cancer metastasis reviews. 2014,33(1):1-16.

26. Yang J, Niu H, Chen X. GATA1-Activated HNF1A-AS1 Facilitates the Progression of Triple-Negative Breast Cancer via Sponging miR-32-5p to Upregulate RNF38. Cancer management and research. 2021,13:1357-69.

27. Wang $C$, Mou L, Chai H, Wang F, Yin Y, Zhang X. Long noncoding RNA HNF1A-AS1 promotes hepatocellular carcinoma cell proliferation by repressing NKD1 and P21 expression. Biomedicine \& pharmacotherapy = Biomedecine \& pharmacotherapie. 2017,89:926-32.

28. Bi Y, Mao Y, Su Z, Du J, Ye L, Xu F. Long noncoding RNA HNF1A-AS1 regulates proliferation and apoptosis of glioma through activation of the JNK signaling pathway via miR-363-3p/MAP2K4. Journal of cellular physiology. 2021,236(2):1068-82.

29. Lou P, Ding T, Zhan X. Long Noncoding RNA HNF1A-AS1 Regulates Osteosarcoma Advancement Through Modulating the miR-32-5p/HMGB1 Axis. Cancer biotherapy \& radiopharmaceuticals. 2021,36(4):371-81.

30. Wang Z, Liu L, Du Y, Mi Y, Wang L. The HNF1A-AS1/miR-92a-3p axis affects the radiosensitivity of non-small cell lung cancer by competitively regulating the JNK pathway. Cell biology and toxicology. 2021,37(5):715-729.

31. Liu Z, Li H, Fan S, Lin H, Lian W. STAT3-induced upregulation of long noncoding RNA HNF1A-AS1 promotes the progression of oral squamous cell carcinoma via activating Notch signaling pathway. 
Cancer biology \& therapy. 2019,20(4):444-53.

32. Liu H, Ma R, Lv B, Zhang H, Shi D, Guo X, et al. LncRNA-HNF1A-AS1 functions as a competing endogenous RNA to activate PI3K/AKT signalling pathway by sponging miR-30b-3p in gastric cancer. British journal of cancer. 2020,122(12):1825-36.

33. Feng Z, Wang B. Long noncoding RNA HNF1A-AS1 promotes cell viability and migration in human bladder cancer. Oncology letters. 2018,15(4):4535-40.

34. Yang $X$, Song J, Cheng Y, Wu W, Bhagat T, Yu Y, et al. Long noncoding RNA HNF1A-AS1 regulates proliferation and migration in oesophageal adenocarcinoma cells. Gut. 2014,63(6):881-90.

\section{Figures}
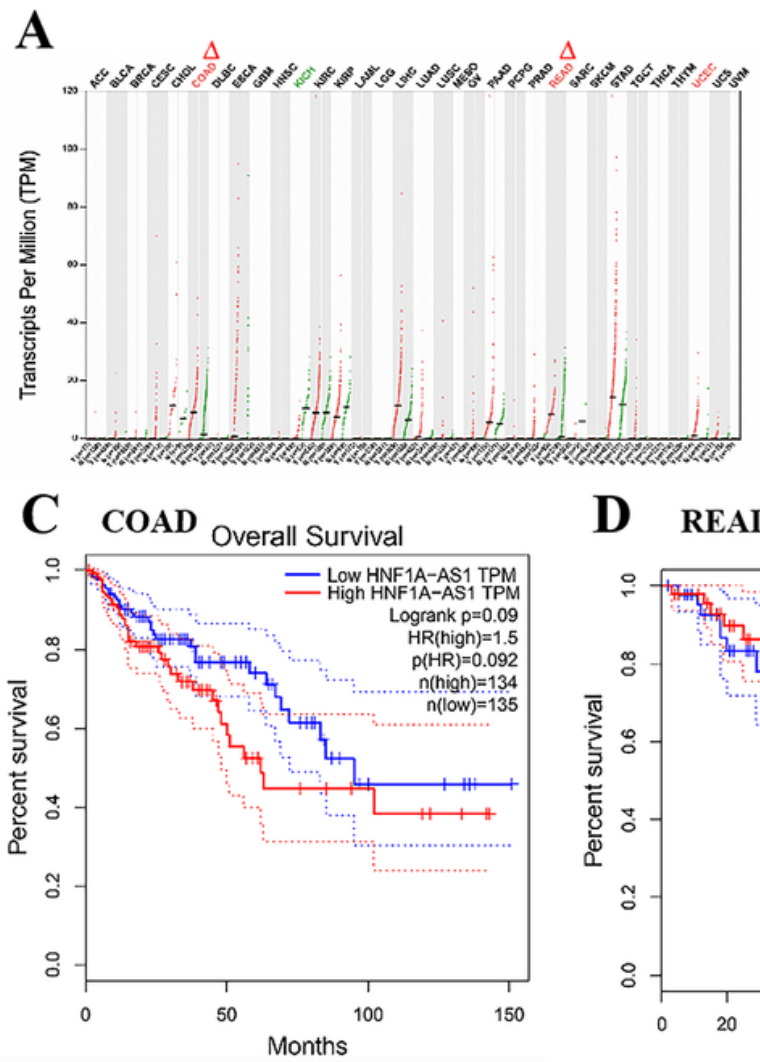

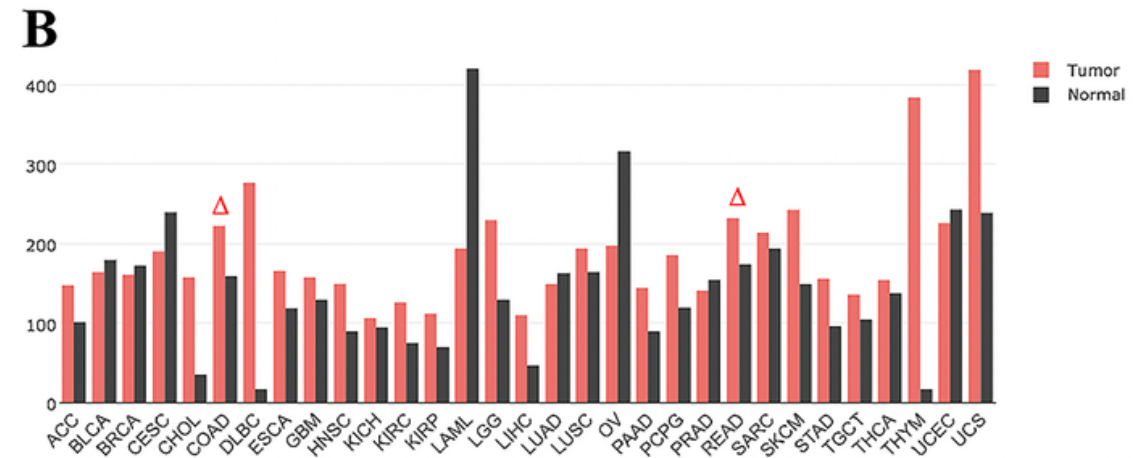

D READ

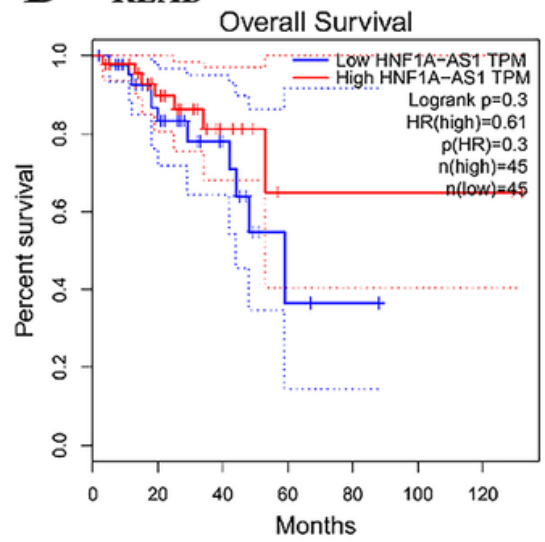

E COAD

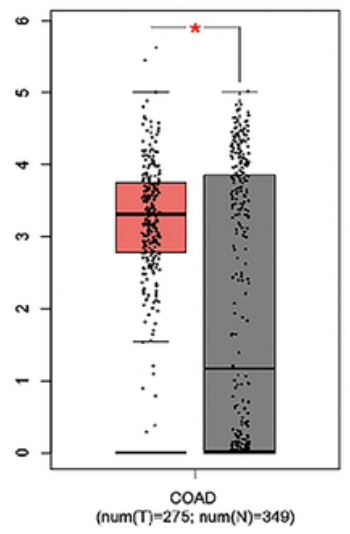

F READ

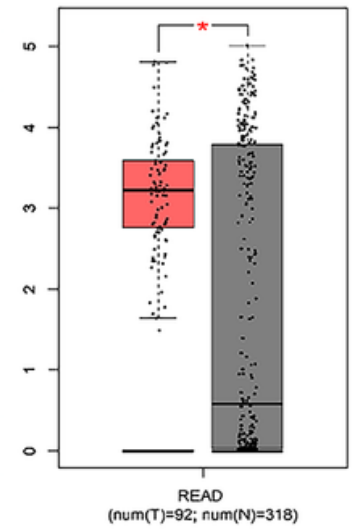

\section{Figure 1}

LncRNA HNF1A-AS1 is up-regulated and association with OS. A, B The expression of HNF1A-AS1 in disparate types of cancers were exhibited with red dots and in para-cancer is shown with green dots. Data derived from TCGA database portal in GEPIA. Full name of different tumors reference supplementary materials. C Kaplan-Meier analysis of colon cancer patients with high $(n=134)$ or low $(n=135)$ HNF1A-AS1 levels. Statistical analysis was performed by log-rank test. D Kaplan-Meier analysis of rectal cancer patients with high $(n=45)$ or low $(n=45)$ HNF1A-AS1 levels. Statistical analysis was performed by log-rank test. E The expression of HNF1A-AS1 is up-regulated in colon cancer compared with para-cancer tissues 
$(P<0.05)$. $\mathbf{F}$ The expression of HNF1A-AS1 is up-regulated in rectal cancer compared with the para-cancer tissues $(P<0.05)$.
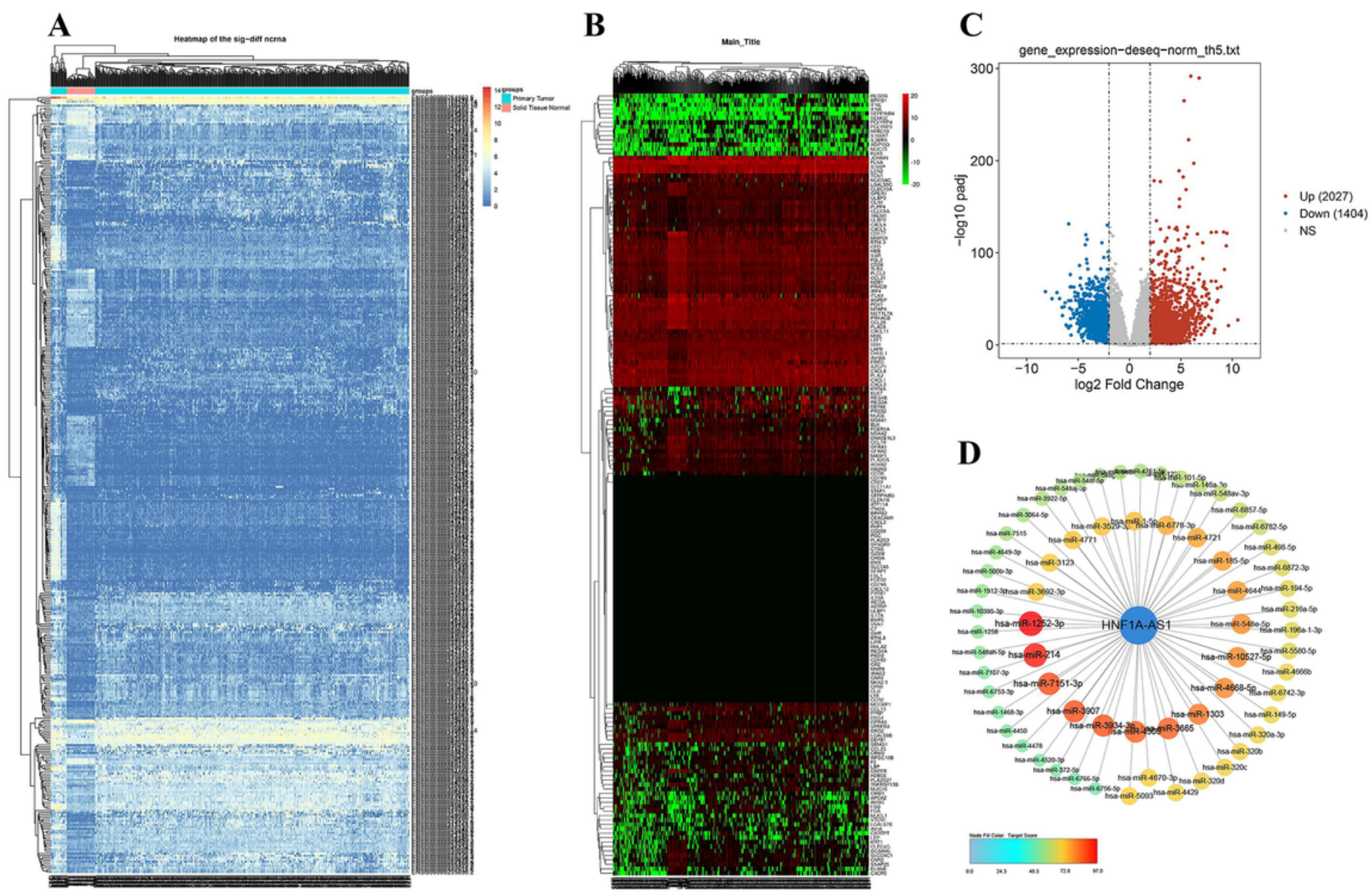

\section{Figure 2}

The differential expression of AS1 in tumor and adjacent tissues and its correlation with differential expression of miRNAs. A Hierarchical cluster analysis of HNF1A-AS1 expression in 477 CRC and 41 adjacent tissues, B Hierarchical cluster of 125 immune related gene expression differences associated with AS1, C Volcano plot of differentially expressed miRNAs(DEMs), D Combined with TargetScan data, HNF1A-AS1 potential associated with DEMs. 
A

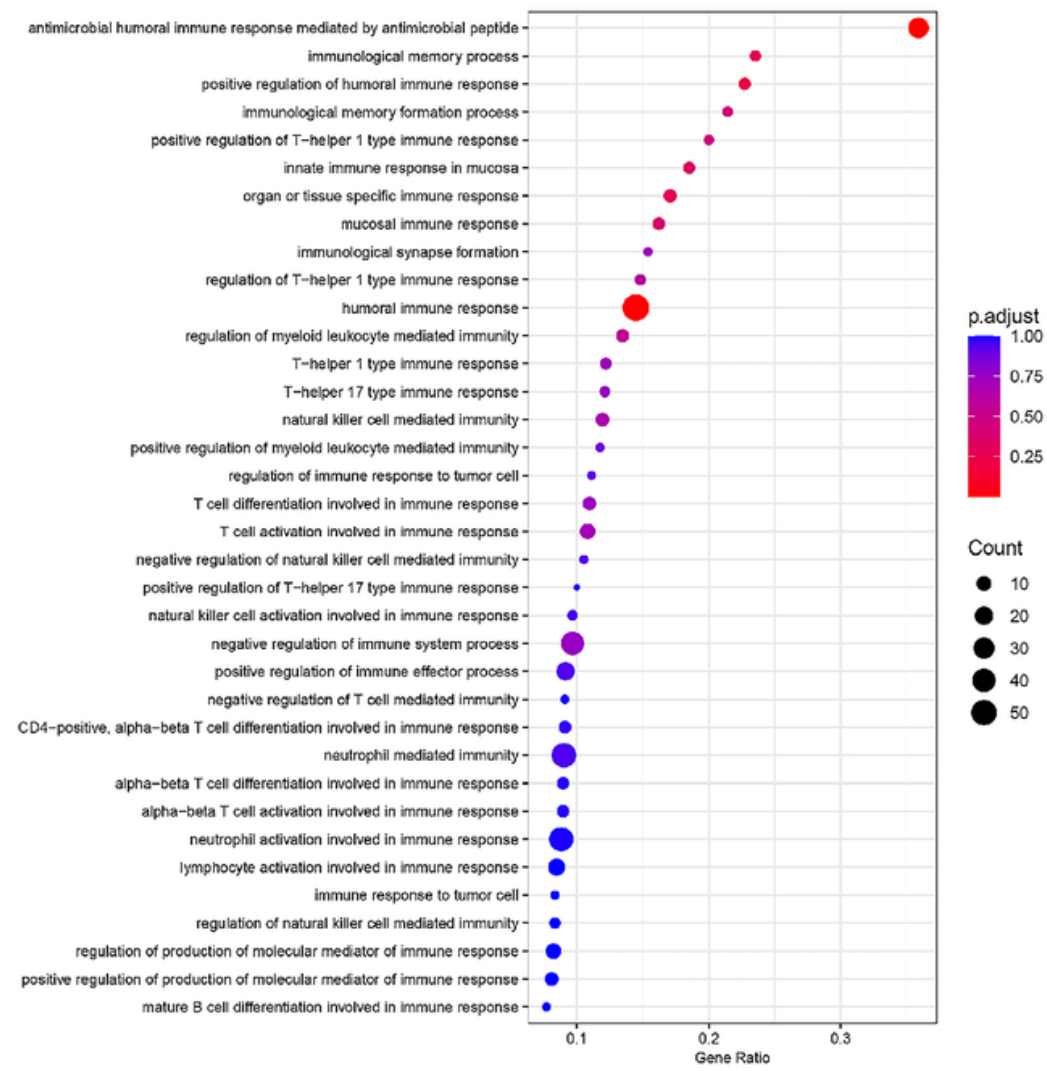

B

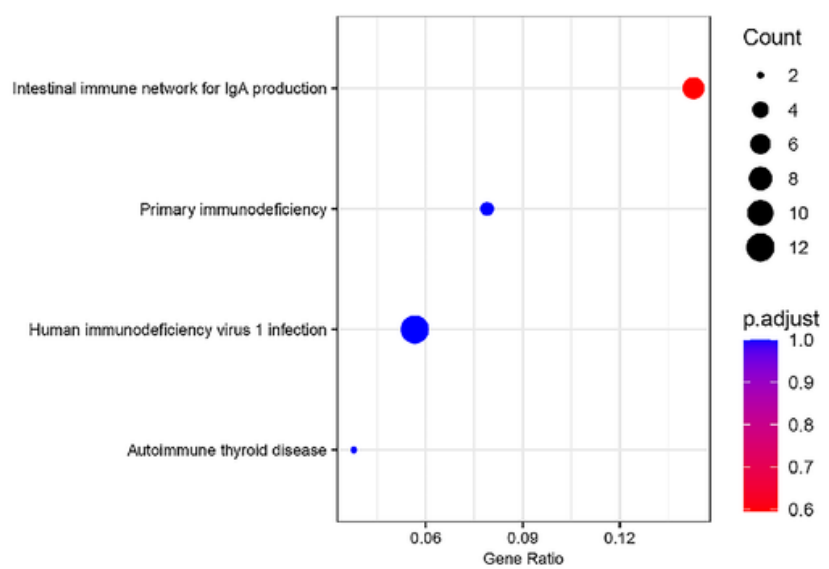

\section{Figure 3}

GO annotation and KEGG pathway enrichment analysis. A A biological process rich in 125 genes related to immunity. B KEGG pathway enriched by 125 immune-related genes.

A
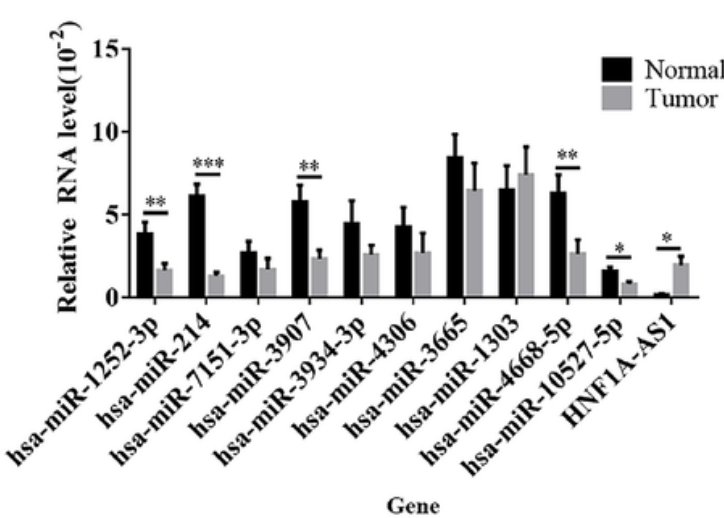

D

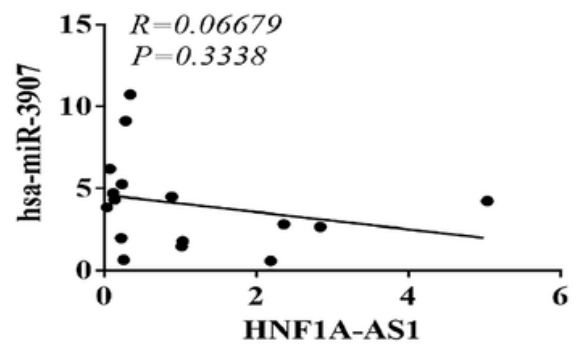

B

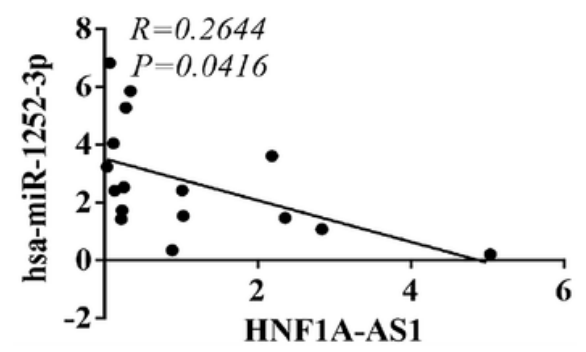

C

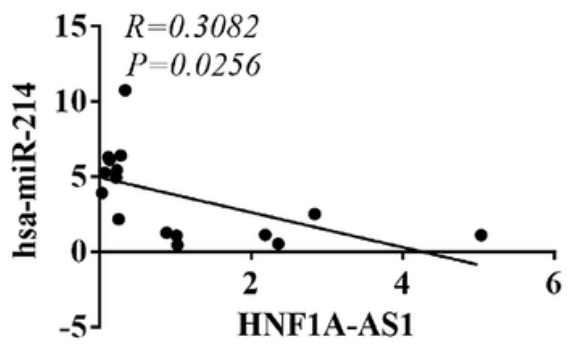

E

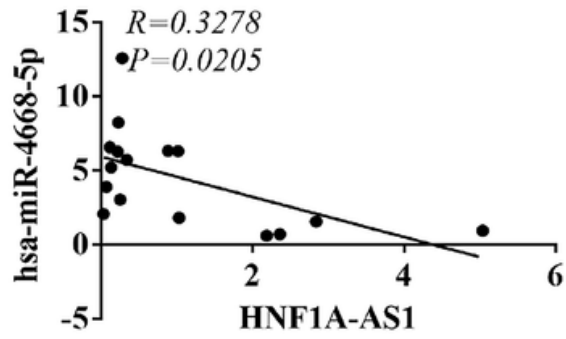

F

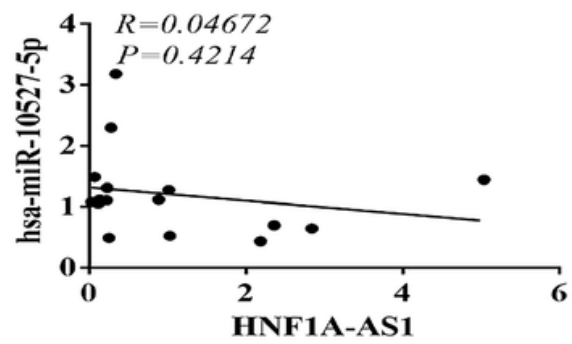


Figure 4

Expression and correlation of HNF1A-AS1 and related miRNAs. A mRNA expression of HNF1A-AS1 and related miRNAs in tumor and normal tissues. $\mathbf{B}$ The correlation analysis between hsa-miR-1252-3p and TMEM220-AS1 expression in CRC tissues. C The correlation analysis between hsa-miR-214 and TMEM220-AS1 expression in CRC tissues. D The correlation analysis between hsa-miR-3907 and TMEM220-AS1 expression in CRC tissues. E The correlation analysis between hsa-miR-4668-5p and TMEM220-AS1 expression in CRC tissues. F The correlation analysis between hsa-miR-10527-5p and TMEM220-AS1 expression in CRC tissues.

A

hsa-miR-1252-3p (Target gene)

\section{3}

88

33
98

104

Immune-related differential genes

hsa-miR-214 (Target gene)

B

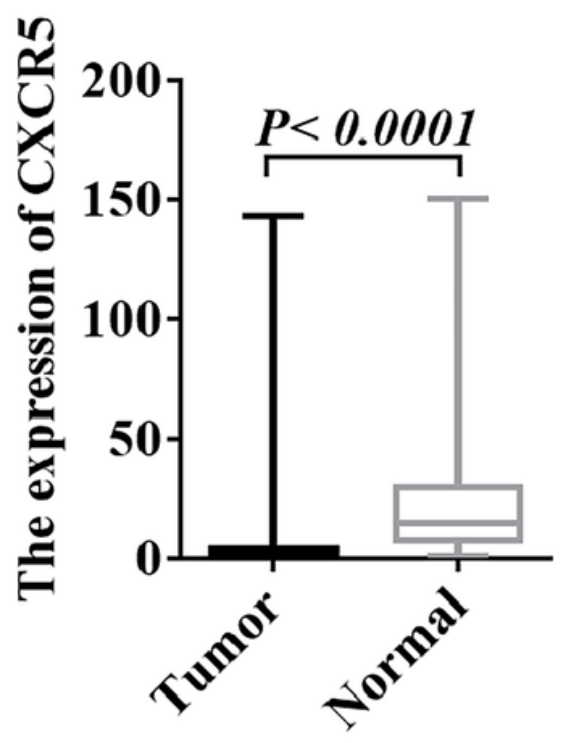

C

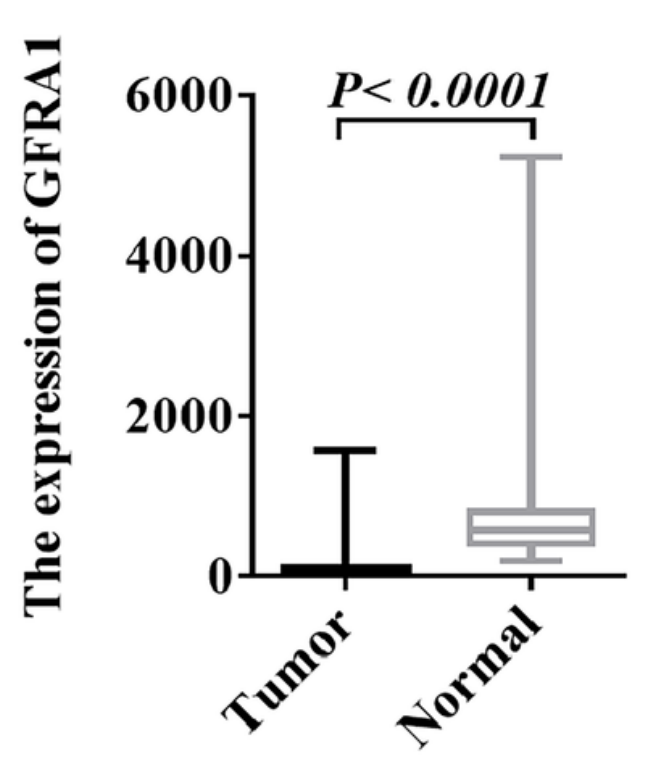

D

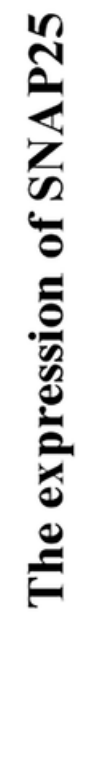

E

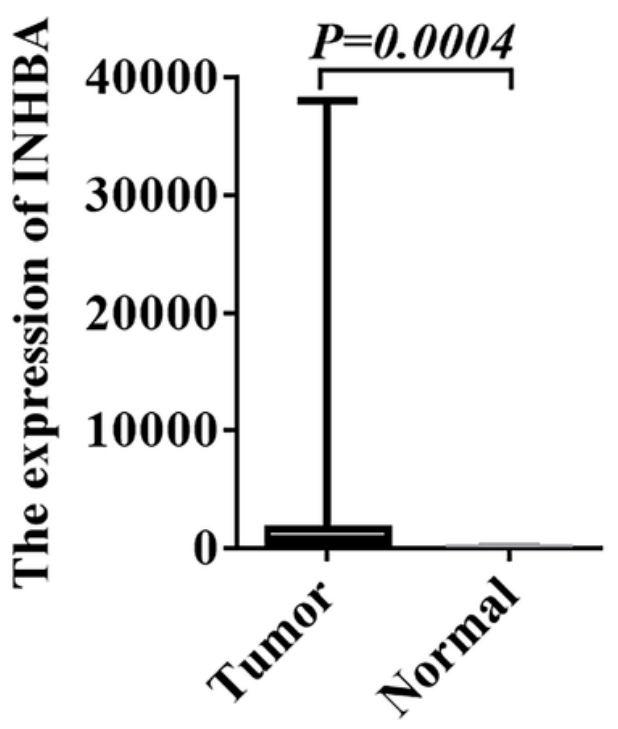

Figure 5 
Expression levels of target genes of hsa-miR-214 in CRC and normal tissues. A The miRDB, miRWalk, and TargetScan databases were utilized to predict the target genes of hsa-miR-214. B Expression of CXCR5 gene in CRC and normal tissues. C Expression of GFRA1 gene in CRC and normal tissues. D Expression of SNAP25 gene in CRC and normal tissues. E Expression of INHBA gene in CRC and normal tissues.

\section{A}

Binding Site of hsa-miR-214-5p on INHBA:

\begin{tabular}{|c|c|c|c|c|c|c|}
\hline Show $10 \vee$ entries & & & & & Search: & \\
\hline BindingSite & $\uparrow$ & Class & Alignment & Th & AgoExpNum $1 \downarrow$ & CleaveExpNum \\
\hline chr7:41728697-41728717[-] & $\uparrow$ & 7 mer-A1 & 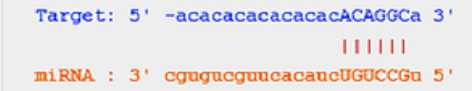 & $\uparrow$ & 1 & 0 \\
\hline
\end{tabular}

B

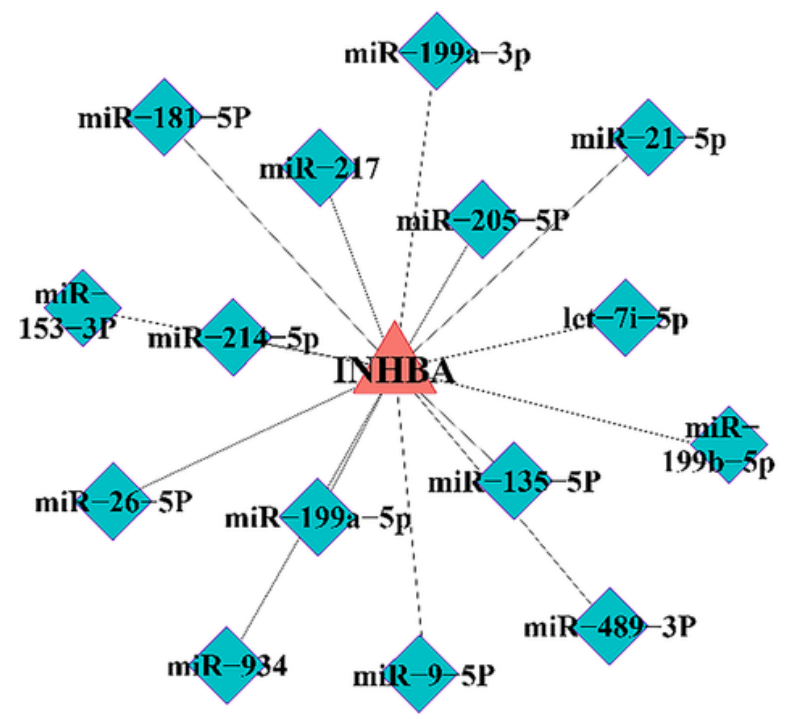

C

hsa-miR-214-5p vs. INHBA, 450 samples (COAD) Data Source: starBase v3.0 project

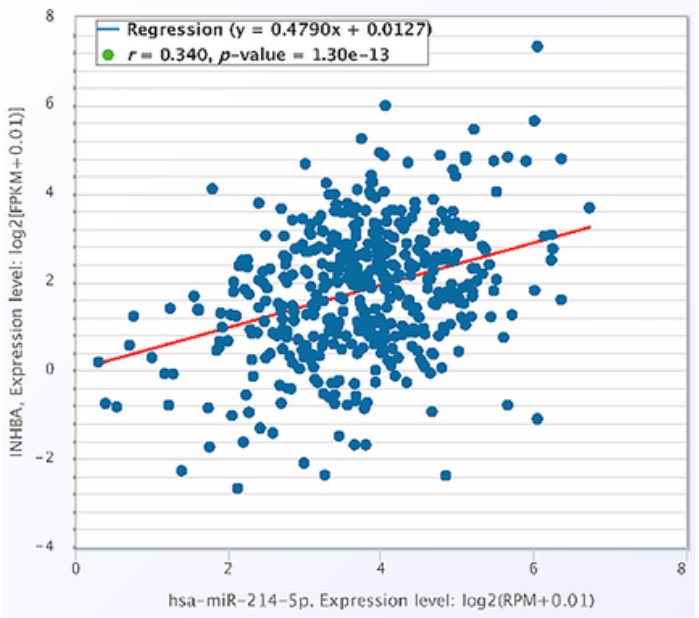

D

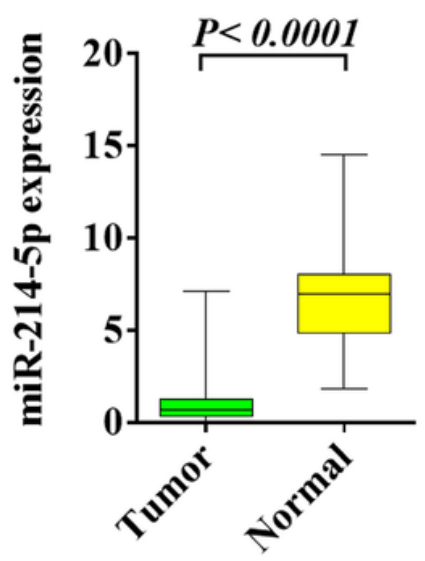

\section{Figure 6}

Bioinformatics analysis of the upstream and downstream regulatory relationship between miR-214-5p and INHBA and their expression in CRC. A The binding site of miR-214-5p in INHBA was identified in the starBase. B The upstream and downstream regulatory relationship between miR-214-5p and INHBA was analyzed by enrichment. C miRNA-214-5p is positively correlated with INHBA in colon cancer patients based on TCGA database. D The expression of miRNA-214-5p in CRC and adjacent tissues was analyzed by TCGA database.

\section{Figure 7}

Bioinformatics analysis of the expression of INHBA in CRC and its correlation with the prognosis. A, B The expression of INHBA in CRC and adjacent tissues was analyzed by TCGA database. C The 
expression of INHBA in different T stages of CRC was analyzed by TCGA database. D The expression of INHBA in different $\mathrm{N}$ stages of CRC was analyzed by TCGA database. $\mathbf{E}$ The expression of INHBA in different $M$ stages of CRC was analyzed by TCGA database. F OS analysis corresponding to INHBA expression in CRC patients in GEPIA database. G DFS analysis corresponding to INHBA expression in CRC patients in GEPIA database. $\mathbf{H}$ Prognostic risk scoring model of CRC based on INHBA gene.

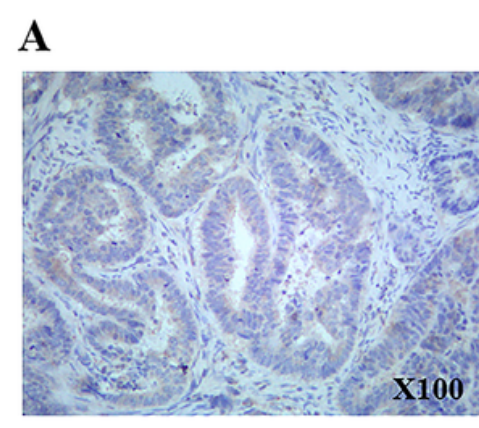

$\mathbf{E}$

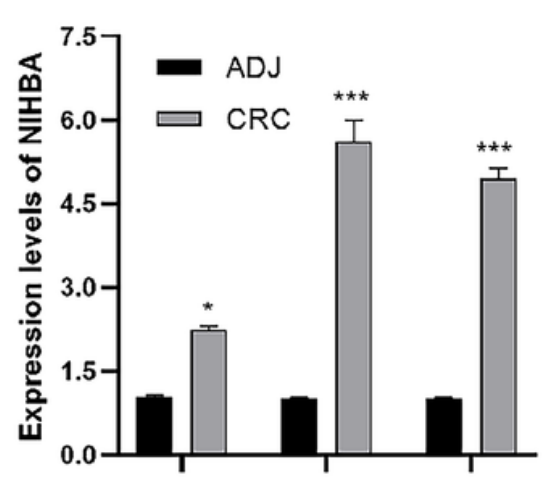

B

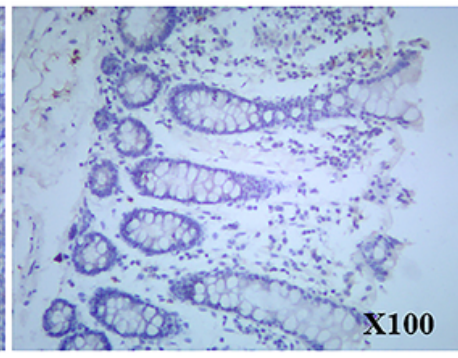

F

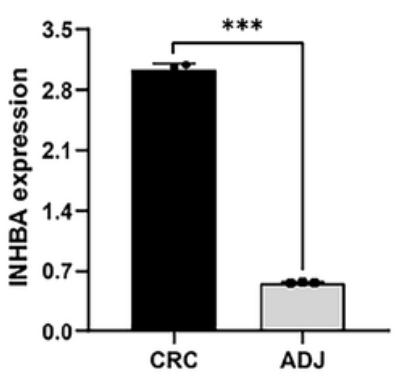

GAPDH

INHBA
C
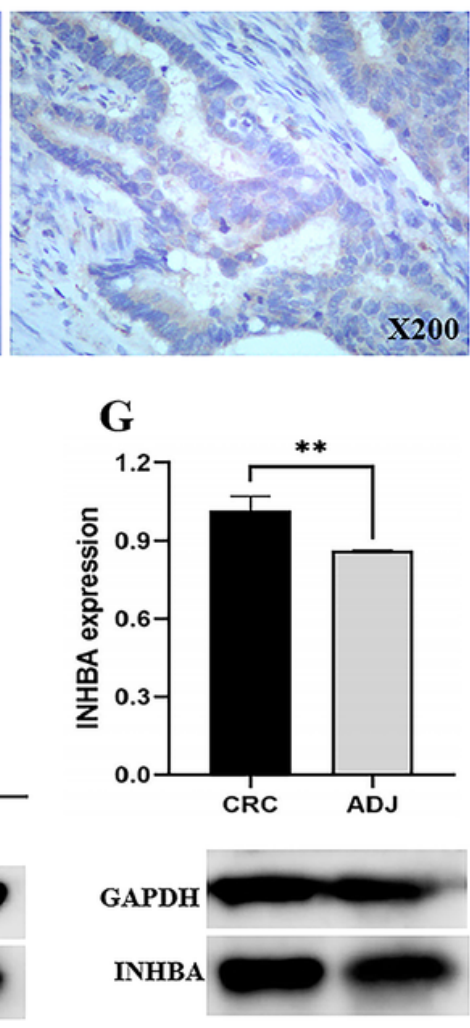

D

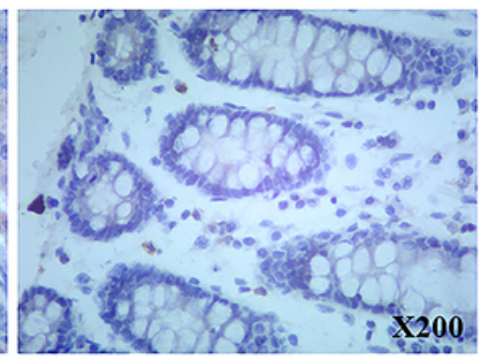

$\mathbf{H}$

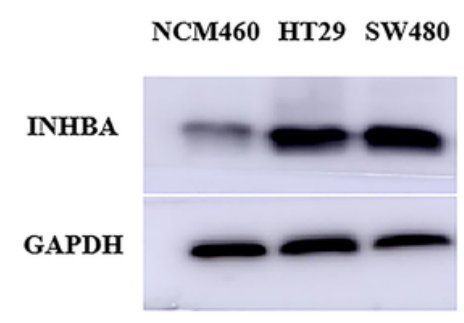

\section{Figure 8}

Expression of INHBA in CRC tissues and cell lines. The histopathological sections of INHBA showed typical IHC staining (A,C: colon cancer tissues, B,D: adjacent tissues). E RT-qPCR showed the expression of INHBA mRNA in CRC and adjacent tissues. F,G Western Blot detection showed the expression of INHBA protein in CRC. $\mathbf{H}$ Western Blot assay showed the expression of INHBA protein in CRC cell lines.

\section{Supplementary Files}

This is a list of supplementary files associated with this preprint. Click to download.

- Figure8WBoriginalblotsgels.zip 